\title{
Prion Interaction with Normal Protein in Topological Changing Secondary Structure to Aggregation
}

Yao Yao*

Department of Basic Medicine, Jiangsu Vocational College of Medicine, Yancheng 224005, China. E-mail: yazh807@163.com

Abstract: Prion is a protein smaller than virus and it infects host in the absence of nucleic acid. The secondary structure of protein folds incorrectly from $\alpha$-helices to $\beta$-sheets through breaking and re-formation of hydrogen bond. Structural analogy of $\alpha$-helix and DNA double helix and comparing differences between $\alpha$-helix and $\beta$-sheet show prion's infectivity and propagation. Aggregates of dimers and polymers generate $\beta$-amyloid fibril in Alzheimer's disease.

Keywords: Prion; $\alpha$-helix; $\beta$-sheet; Hydrogen Bond; Aggregation

\section{Introduction}

Prions, also known as protein infectious factors or infectious proteins, are small non-immune hydrophobic proteins that can infect animals. They replicate in host cells. Prions are as infectious and pathogenic as conventional viruses but much smaller than the smallest known conventional viruses (about 30-50 nm). The structure of virus particle is not observed under electron microscope. Prion protein is the coding product of human and animal normal cell genes and its human gene is located in the short arm of chromosome 20. It causes degeneration of the central nervous system in humans and livestock. There are four kinds of prion diseases in human: Kuru's disease, K-ya's syndrome, Germain's syndrome and fatal familial insomnia. It is also linked to Alzheimer's disease ${ }^{[1]}$.

Prion diseases in mammals are caused by a conformational transition of the cellular protein from its native conformation ( $\mathrm{PrPc}$ ) to a pathological isoform called "prion protein scrapie" $(\mathrm{PrPSc})^{[2]}$. There is no difference in primary structure between PrPc and PrPSc.
As to secondary structure, $42 \%$ are $\alpha$-helices and $3 \%$ are $\beta$-folds in $\mathrm{PrPc}, 3 \%$ are $\alpha$-helices and $42 \%$ are $\beta$-folds in PrPSc. There is only PrPc in the brain of normal animals and no PrPSc. The transition from $\alpha$-helix to $\beta$-sheet of PrPc and PrPSc means a lot.

\section{Materials and methods}

\subsection{Structural analogy of $\alpha$-helix and DNA double helix}

The basic unit of protein or polypeptide is $20 \mathrm{kinds}$ of amino acids. Secondary structure of a protein refers to specific conformation of polypeptide main chain, which does not involve the position of amino acid side chains. The forms of secondary structure include $\alpha$-helix, $\beta$-sheet, $\beta$-corner and random curl. The secondary structure of a polypeptide chain is a combination of these different types of conformation. The main force to maintain the secondary structure is hydrogen bond.

Each circle of a right-handed $\alpha$-helix has 3.6 residues on average. The $\mathrm{H}$ that covalent binds to $\mathrm{N}$ at the first amino acid and the $\mathrm{O}$ at the fourth amino acid

\footnotetext{
Copyright (C) 2020 Yao Yao

doi: 10.18686/aem.v9i2.167

This is an open-access article distributed under the terms of the Creative Commons Attribution Non-Commercial License

(http://creativecommons.org/licenses/by-nc/4.0/), which permits unrestricted non-commercial use, distribution, and reproduction in any medium,

provided the original work is properly cited.
} 
forms a hydrogen bond (Figure 1A).

The molecular weight of nucleic acid ranges from 100,000 to $1,000,000$ and the average molecular weight of a single nucleotide is 330 . The molecular weight of protein ranges from 10,000 to $1,000,000$ and the molecular weight of a single amino acid ranges from 90 to 200 , and the average molecular weight is 128 . Prions are protein particles with molecular weight of 27,000-30,000.

A

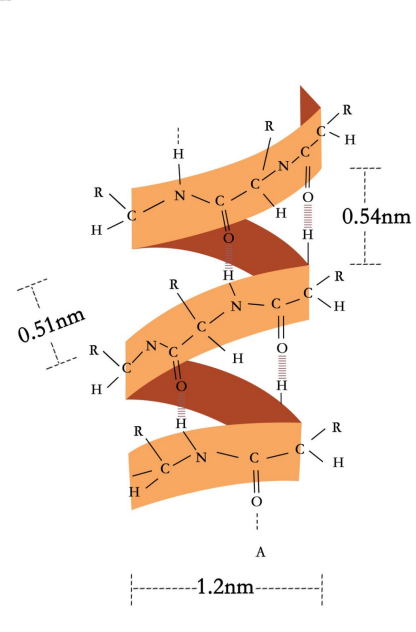

In DNA double helix, there are two types of hydrogen bond. Both $\mathrm{H}$ covalent bind to $\mathrm{N}$. One type is formed by $\mathrm{O}$ and $\mathrm{H}$ and the average energy is $8 \mathrm{~kJ} / \mathrm{mol}$ and the other type is formed by $\mathrm{N}$ and $\mathrm{H}$ and the average energy is $13 \mathrm{~kJ} / \mathrm{mol}$ (Figure 1B).

Comparing with DNA double helix, the gap between $\alpha$-helix circles is wide enough to accommodate prion proximity and interaction.

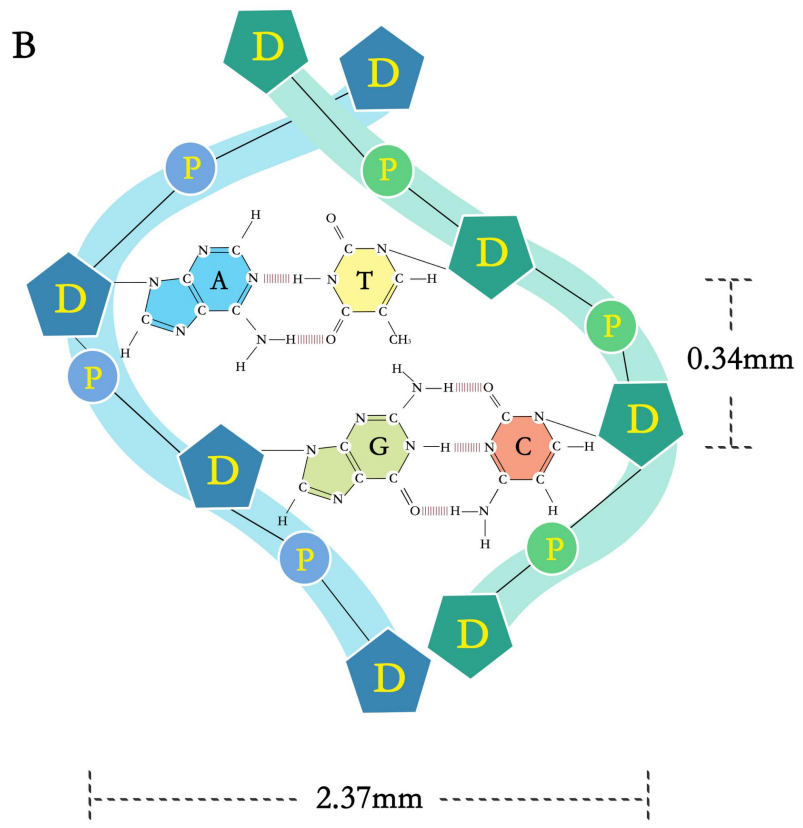

Figure 1. The hydrogen bonds of $\alpha$-helix and DNA double helix.

A $\alpha$-helix. A right-handed helix formed by a peptide chain. The $\mathrm{H}$ that covalent binds to $\mathrm{N}$ at the first amino acid and the $\mathrm{O}$ at the fourth amino acid forms a hydrogen bond. B, DNA double helix. Two kinds of hydrogen bonds in base pairs: one formed by $\mathrm{N}$ and $\mathrm{H}$ and one formed by $\mathrm{O}$ and $\mathrm{H}$. Both of $\mathrm{H}$ covalently bound N.

\subsection{Comparison of two secondary structures of protein: $\alpha$-helix and $\beta$-sheet}

While $\alpha$-helix is formed by a single strand peptide chain, $\beta$-sheet is a plane structure formed by two or more peptide segments. These peptide chains are parallel or antiparallel with hydrogen bonds formed by $\mathrm{H}$ and $\mathrm{O}$ on opposite amino acids.

Topological changing secondary structure starts from interaction of PrPSc with PrPc. PrPc hydrogen bonds at $\alpha$-helix break down and the peptide chain strand extends and binds to PrPSc with new hydrogen bonds (Figure 2). $\beta$-sheet formation ends with aggregation of multiple peptide segments. The process undergoes multiple hydrogen bond breaking and re-formation. 


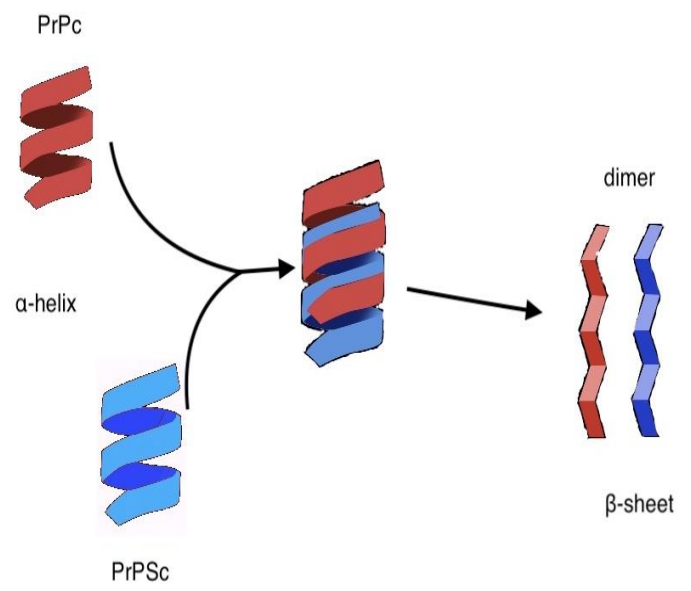

Figure 2. Prion interaction with normal protein in transformation from $\alpha$-helix to $\beta$-sheet.

\subsection{Aggregation and disaggregation of prion monomers}

Aggregates consist of groups of misfolding proteins and every discrete size should be larger than the critical nucleus $\operatorname{size}^{[3]}$. The aggregation and disaggregation process is dynamic. When aggregation overcome, it is beneficial to folding.

\section{Results}

\subsection{Topological change of transformation of $\alpha$-helix to $\beta$-sheet}

If the hydrogen bonds maintaining $\alpha$-helix are broken, the peptide chain can combine with another peptide chain to form a dimer. Through structural topological change, the original right-hand $\alpha$-helices, with side chain groups of amino acid residue extend to the outside, and it forms a $\beta$-sheet conformation, with side chains of amino acid residue distributed above and below the lamella.

\section{2 $\beta$-sheet forms and monomers aggregate}

Small polypeptide chains in $\beta$-sheet are connected by hydrogen bonds. In $\beta$-sheet, hydrogen bonds are formed by $\mathrm{H}$ and $\mathrm{O}$. It may also be formed by $\mathrm{H}$ and $\mathrm{N}$. Combination of peptide segments. Two hydrogen bonds make structure have more stability, greater diversity and more possibilities. Thus, monomers aggregate to oligomers and polymers (Figure 3 ).

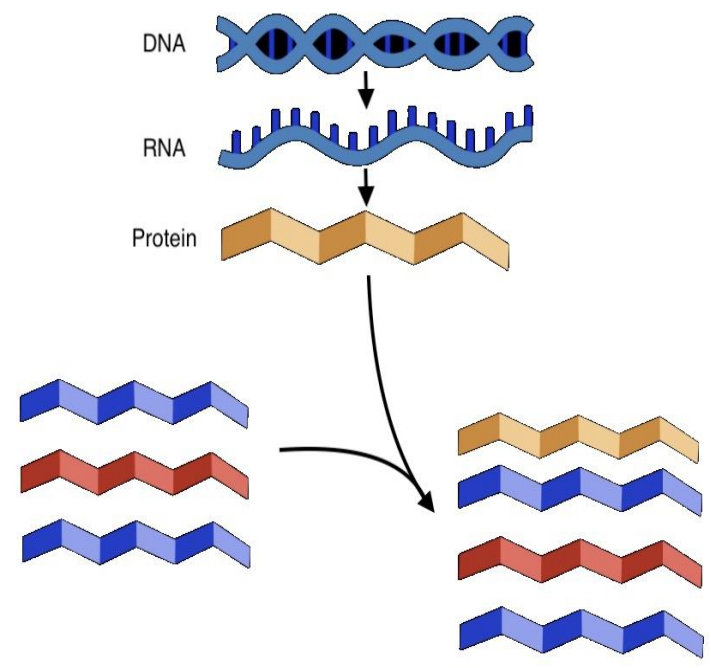

Figure 3. Misfolding proteins induced fit to aggregate.

The insoluble protein is resistant to proteolysis. The protein particles have such strong resistance to various physical and chemical actions that cause diseases of typical features, including extensive cavernous degeneration, amyloid deposition and neurodegeneration, and it even leads the host to death. 


\subsection{Protein transformation causes protein denaturation}

Theoretically, the smallest PrPSc aggregate is a dimmer containing disulfide bond and PrPSc dimerization may contribute to prion diseases. Fatal familial insomnia brain biopsies formed more $\operatorname{PrP}$ dimmers ${ }^{[4]}$. Through the increase of molecular size and hydrophobic amino acid number, solubility of protein in water decrease, generating precipitation. In this case, as under action of some physical and chemical factors, the spatial structural change leads to protein denaturing, that is, the change of physical and chemical properties and the loss of biological activity. Therefore, changing protein secondary structure may be fatal. The transformation of $\alpha$-helix and $\beta$-sheet deprives the original properties and functions.

\section{Discussion}

\subsection{The effect of hydrogen bond on secondary structural topological change}

While DNA double chain is stable as genetic material for four bases in the formation as $\mathrm{A}$ and $\mathrm{T}$ connected by two hydrogen bonds and $\mathrm{C}$ and $\mathrm{G}$ connected by three hydrogen bonds, the $\mathrm{H}$ and $\mathrm{O}$ of amino acids form hydrogen bonds of protein secondary structure. The two atom types, $\mathrm{H}$ and $\mathrm{O}$, determines whether the secondary structure is $\alpha$-helix or $\beta$-sheet, which explains why proportion of $\alpha$-helix and $\beta$-sheet converse totally in prions.

\subsection{Independent propagation and pathogenicity based on topological changing secondary structure of normal protein as the 'protein-only hypothesis' ${ }^{[5]}$}

If PrPSc exists for some time, it may interact with other normal proteins and fold incorrectly. The topology structure changes through breaking and re-formation of hydrogen bond, so the types of protein and amino acid sequence are less important. The misfolding protein propagate and enlarge layer by layer to a huge spherical fourth structure. Chemical bonds on a layer may break down and chemical bonds between two layers may be formed. Due to changing spatial structure that leads to dysfunction, prions need not reverse to generate nucleic acids to express in host cells under catalysis of reverse transcriptase as ordinary viruses.

\section{3 'Protein-only' refractory diseases}

Though protein translation codons containing genetic information are universal, mismatched proteins all function abnormally. Aggregation of proteins to a certain extent inevitably generate amyloid- $\beta$ and irreversible dysfunction of nervous system that have observed in the brain of Alzheimer's patient. It may signify that these diseases are less correlate with heredity and environmental related.

\section{Conclusion}

Prion topological changing protein secondary structure leads to protein dysfunction and it causes several diseases. From $\alpha$-helix to $\beta$-sheet through breaking and re-formation of hydrogen bond, proteins aggregate and prion propagates in the absence of nucleic acid. Protein structural topological changing displays an important role in prion infectivity indeed.

Compliance with Ethical Standards: This study wasn't funded.

\section{Conflict of Interest}

The author declares that there is no conflict of interest.

\section{References}

1. John A, Myers RR, Fernandez FP, et al. Effects of mutations on human prion protein unfolding. Biophysical Journal 2020; 118(3): 372a. doi: 10.1016/j.bpj.2019.11.2130.

2. Pravas KB, Jiang Y, Adriano A, et al. Transition of the prion protein from a structured cellular form (PrPC) to the infectious scrapie agent (PrPSc). Protein Science 2019; 28(12): 2055-2063.

3. Banwarth-Kuhn M, Sindi S. How and why to build a mathematical model: A case study using prion aggregation. Journal of Biological Chemistry 2020; 295(15): 5022-5035. doi: 10.1074/jbc.REV119.009851.

4. Gao Z, Shi J, Cai L, et al. Prion dimer is heterogenous and is modulated by multiple negative and positive motifs. Biochemical \& Biophysical Research Communications 2019; 509(2): 570-576. doi: 10.1016/j.bbrc.2018.12.113.

5. Ma J, Wang F. Prion disease and the 'protein-only hypothesis'. Essays in Biochemistry 2014; 56: 181-191. doi: 10.1042/bse0560181. 